\title{
CORRESPONDENCE
}

\section{Macrophage subsets at the maternal-fetal interface}

\author{
Xiangxiang Jiang $\mathbb{D}^{1,2}$ and Hongmei Wang $\mathbb{D}^{1,2,3}$ \\ Cellular \& Molecular Immunology (2020) 17:889-891; https://doi.org/10.1038/s41423-020-0435-6
}

Decidual macrophages account for $10-20 \%$ of decidual immune cells during early human pregnancy and have been reported to play roles in tissue remodeling, debris clearance, and parturition. ${ }^{1}$ Investigators consistently consider decidual macrophages a homogenous population in studies. ${ }^{2}$ By using CCR2 and CD11C, we divided decidual macrophages into three distinct subsets during early human pregnancy. ${ }^{3}$ CCR2-CD11cLO macrophages were the most abundant ( 80\%) macrophage subset and were evenly distributed in the decidua. CCR2 $+\mathrm{CD} 11 \mathrm{cH}$ macrophages (10-15\%) were proinflammatory and mainly distributed proximal to extravillous trophoblast cells (EVTs). Remarkably, a novel decidual macrophage population, the heme oxygenase-1 positive $(\mathrm{HMOX} 1+)$ macrophage subset (CCR2-CD11cHI), which was the lowest ( 5\%) of the three populations studied, was identified and found to be localized strictly proximal to EVTs. Here, we first report our experimental progress on decidual macrophage subsets and then discuss important studies related to the two $\mathrm{CD} 11 \mathrm{cH}$ macrophage subsets located proximal to EVTs.

The three macrophage subsets not only expressed different levels of CCR2 and CD11c but also exhibited different expression levels of CD45 and CD14 by flow cytometry analysis (Fig. 1a). The expression levels of CD45 and CD14 paralleled each other from low to high levels in CCR2-CD11CLO macrophages. The CCR2$\mathrm{CD} 11 \mathrm{cHI}$ macrophage subset had the highest expression levels of CD45 and CD14. The expression level of CD45 in CCR2 + CD11cHI macrophages was close to that in CCR2-CD11cHI macrophages, but this subset had the lowest expression level of CD14 (Fig. 1a). These findings further demonstrated that the three decidual macrophage subsets were truly different from each other and reflected the different roles of these subsets in T-cell activation and the innate immune response., ${ }^{4,5}$

The concepts of $M 1$ and $M 2$ macrophages have been well known in the field of macrophage research. ${ }^{1,6}$ Thus, we sought to elucidate the relationships between these three subsets of decidual macrophages and in vitro-induced M1 or M2 macrophages. M1 and M2 genes were defined based on highly expressed genes that showed at least a two-fold difference between $\mathrm{M} 1$ and $\mathrm{M} 2$ macrophages (Table S1) according to published RNA-Seq data (GSE36952). ${ }^{7}$ Based on the unsupervised clustering results, we found that CCR2 $+\mathrm{CD} 11 \mathrm{cHI}$ macrophages expressed many more M1 macrophage genes and expressed these genes at higher levels than the other two decidual macrophage subsets (Fig. 1b). Furthermore, this CCR2+ macrophage subset clustered with M1 macrophages (Fig. 1C). Considering the proinflammatory properties of this subset, we suggest CCR2 + CD11CHI to be M1-like macrophages in vivo. The two CCR2- macrophage subsets always clustered together, regardless of whether $\mathrm{M} 1$ or $\mathrm{M} 2$ genes were used for evaluation (Fig. 1b-e), suggesting that the CCR2-CD11CLO and CCR2$\mathrm{CD} 11 \mathrm{cHI}$ macrophage subsets share more commonalities with each other than with the CCR2 $+\mathrm{CD} 11 \mathrm{cH}$ subset. Additionally, the expression levels and expression scale of M2 genes were much higher and much more extensive in these two CCR2macrophage subsets than those of $M 1$ genes (Fig. 1b, d). Therefore, we suggest that the CCR2-CD11CLO and CCR2$\mathrm{CD} 11 \mathrm{cHI}$ macrophage subsets are inclined to be antiinflammatory M2-like macrophages; however, these two CCR2subsets did not cluster with M2 macrophages. This might be explained by the discrepancy between the in vitro-induced macrophage type and the in vivo decidual macrophages, and it seemed unlikely that a particular in vivo macrophage population would be fully matched with the in vitro-induced $M 1$ or M2 subtype. ${ }^{6}$ We suggest that because of the high percentage (>80\%) of M2-like CCR2-CD11CLO macrophages at the maternalfetal interface, decidual macrophages are usually considered the M2 type. ${ }^{8}$ Additionally, with the discovery of the three decidual macrophage subsets, investigators could easily detect the percentages of M1- and M2-like macrophages at the maternalfetal interface, which may contribute to the investigation of gestational diseases that correlate with inflammatory factors.

HMOX1 can degrade toxic free heme to execute its antiinflammatory function. ${ }^{9}$ With the discovery of HMOX1+ macrophages, the precise functions of this subset and how effectively HMOX1+ macrophages can protect pregnancies from the inflammatory milieu at the maternal-fetal interface are our next focuses. The discovery of HMOX $1+$ macrophages could also provide insights for many other HMOX1-related studies. In areas where malaria is endemic, sickle hemoglobin helps patients infected by plasmodia survive. ${ }^{10}$ Some studies indicate that sickle hemoglobin easily releases free heme, which could further induce HMOX1 expression in bone marrow- and peripheral bloodderived cells to make an antioxidant and anti-inflammatory milieu to combat plasmodia. ${ }^{10}$ Nonetheless, the precise immune cell types or subsets have not been clarified, and we suggest that HMOX1 + macrophages in other organs may play some role in plasmodia tolerance. HMOX1 can also mediate the therapeutic effects of molecules such as IL-10, which is an anti-inflammatory cytokine, rapamycin and aspirin, functioning as a "therapeutic funnel" ${ }^{11}$ We propose that HMOX1+ macrophages play roles in the course of medical treatment. Although HMOX 1 has been reported to be expressed in human trophoblast cells and plays significant roles during pregnancy, ${ }^{12}$ we have not detected evident and extensive expression of HMOX1 in human trophoblast cells at the maternal-fetal interface. ${ }^{3}$ Therefore, we

\footnotetext{
${ }^{1}$ State Key Laboratory of Stem Cell and Reproductive Biology, Institute of Zoology, Chinese Academy of Sciences, Beijing 100101, China; ${ }^{2}$ Innovation Academy for Stem Cell and Regeneration, Chinese Academy of Sciences, Beijing 100101, China and ${ }^{3}$ Institute of Zoology, University of Chinese Academy of Sciences, Chinese Academy of Sciences, Beijing 100049, China
}

Correspondence: Xiangxiang Jiang (jiangxx@ioz.ac.cn) or Hongmei Wang (wanghm@ioz.ac.cn)

Received: 9 March 2020 Accepted: 3 April 2020

Published online: 7 May 2020 


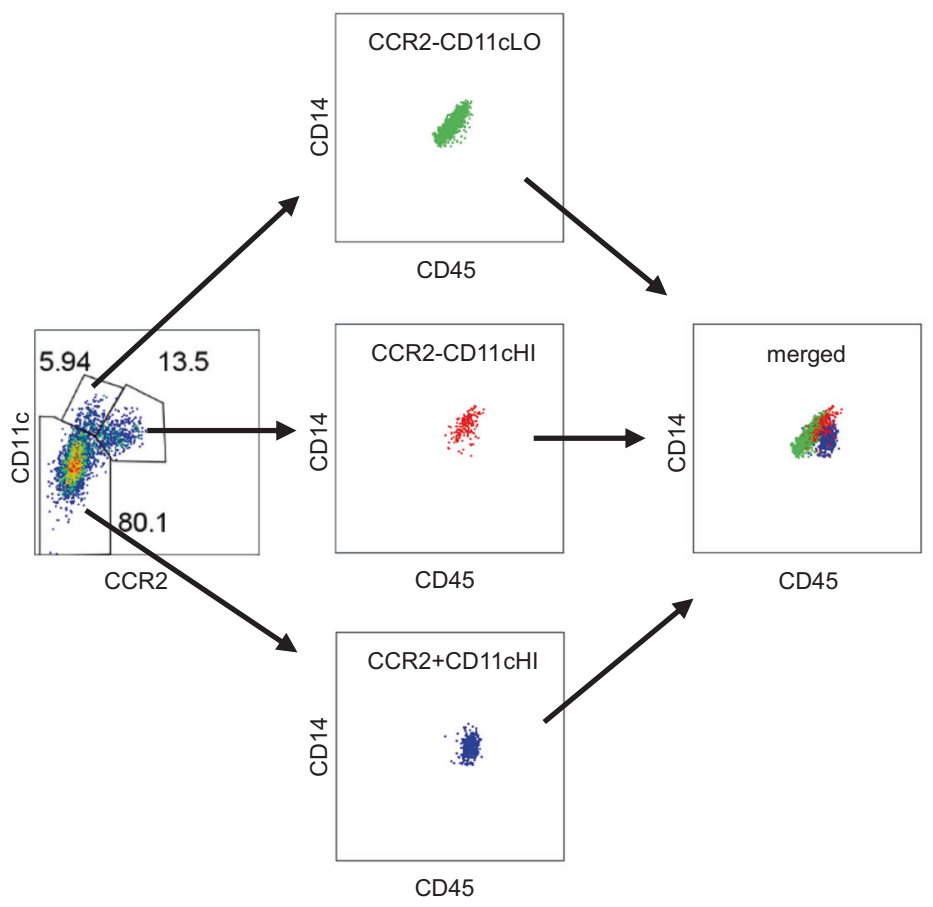

b

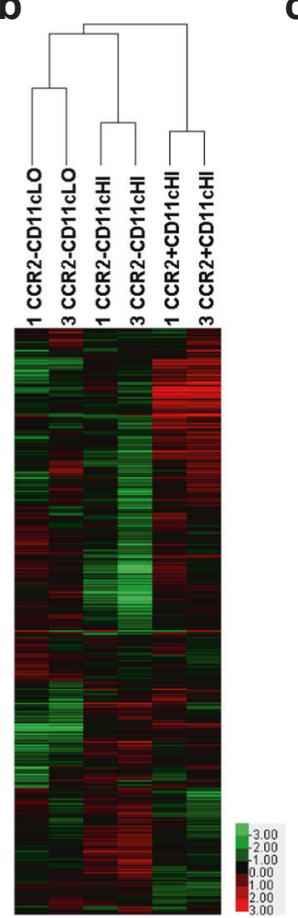

C

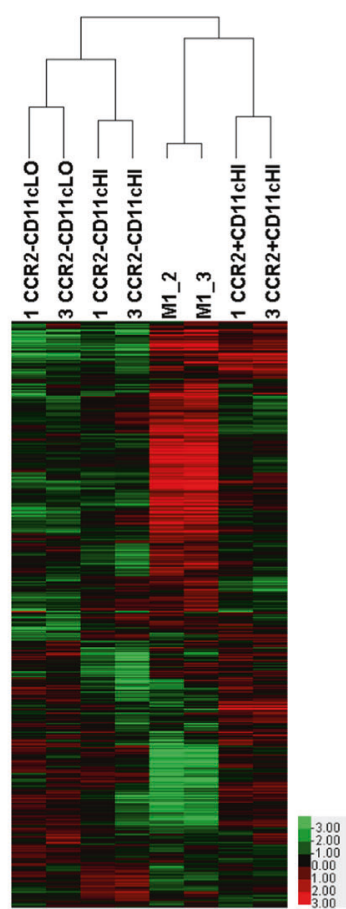

d

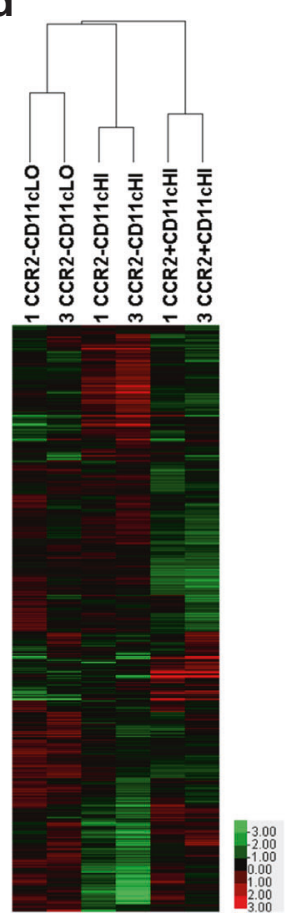

e

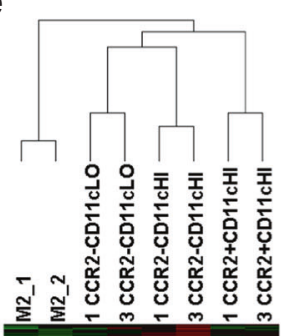

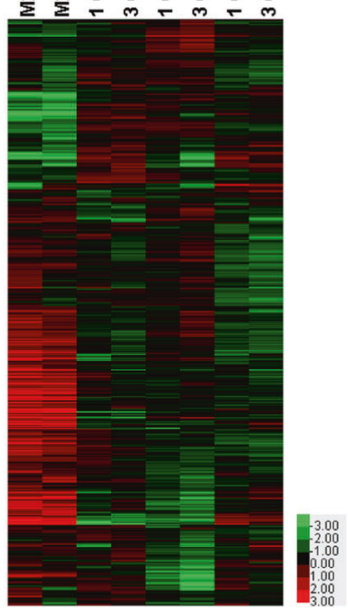

Fig. 1 The expression of CD14, CD45 and M1 and M2 genes in three decidual macrophage subsets. a Flow cytometry analysis of CD14 and CD45 expression in three decidual macrophage subsets. b Heat map analysis of three decidual macrophage subsets with 660 M1 genes. c Heat map analysis of three decidual macrophage subsets and M1 macrophages with 660 M1 genes. d Heat map analysis of three decidual macrophage subsets with 881 M2 genes. e Heat map analysis of three decidual macrophage subsets and M2 macrophages with 881 M2 genes

considered $\mathrm{CCR} 2+\mathrm{CD} 11 \mathrm{cH}$ macrophages to be the cell subset that executes HMOX1 molecular functions at the maternal-fetal interface during pregnancy.

Microbial vertical transmission during human pregnancy has a great influence on baby health. ${ }^{13}$ Human placental syncytiotrophoblast (STB), which covers most of the placental chorion area except the distal ends of chorion anchoring villi, ${ }^{14}$ serves as a strong physical barrier to transplacental transmission of known "TORCH" pathogens, including Toxoplasma gondii, other (Listeria monocytogenes, parvovirus, HIV, etc.), rubella, cytomegalovirus, and herpesvirus. ${ }^{13}$ The loose cell column and EVTs at the distal ends of the anchoring villi in the decidua have been shown to be 
easily penetrated by $T$. gondii, L. monocytogenes, and even Zika virus (ZIKV), especially during early pregnancy. ${ }^{13}$ Recently, a case of neonatal 2019 novel coronavirus disease (COVID-19) in China has been reported, but whether the case is the result of vertical transmission remains to be confirmed. ${ }^{15}$ The two highly phagocytic $\mathrm{CD} 11 \mathrm{cH}$ decidual macrophage subsets, which are localized in front of the distal ends of the anchoring villi, may play significant roles during pathogen vertical transmission, and the details deserve further investigation.

One study reported that mouse lung interstitial macrophages could be divided into three subsets based on the expression levels of CD11C and MHCII, and the identified M3 macrophage subset was CCR2 positive. ${ }^{16}$ In addition, the expression levels of $\mathrm{MHCll}$ molecules, including HLA-DRA, are relatively low in CCR2-CD11cHI decidual macrophages. ${ }^{3}$ These results suggest a general clustering phenomenon widely observed among tissue macrophages in mammalian organs. Decidual macrophages also differentially express other membrane molecules, such as triggering receptor expressed on myeloid cells 1 (TREM1), macrophage mannose receptor 1 (MRC1) and CD209. ${ }^{3}$ These molecules could also be candidate decidual macrophage subset markers. We emphasize the existence of distinct macrophage subsets and their polarized distributions at the maternal-fetal interface, not simply the method used to identify these subsets. To some degree, our findings on macrophage subsets in the human decidua are based on subjective selection of membrane markers. We suggest that single-cell RNA-Seq could help identify macrophage subsets more objectively at the maternal-fetal interface.

Overall, we consider that conclusions drawn from studies in which macrophages are separated into subsets, compared with those drawn from studies considering macrophages a homogenous population, are more convincing and serve as a better guide for the diagnosis and therapy of macrophage-associated diseases. The discovery of macrophage subsets proximal to EVTs may boost decidual macrophage-associated studies.

\section{ACKNOWLEDGEMENTS}

This study was supported by grants from the National Natural Science Foundation of China (31900664 and 81671465) and the Ministry of Science and Technology of the People's Republic of China (2018YFC1004101 and 2017YFC1001401).

\section{ADDITIONAL INFORMATION}

The online version of this article (https://doi.org/10.1038/s41423-020-0435-6) contains supplementary material.

Competing interests: The authors declare no competing interests.

\section{REFERENCES}

1. Yang, F., Zheng, Q. \& Jin, L. Dynamic function and composition changes of immune cells during normal and pathological pregnancy at the maternal-fetal interface. Front Immunol. 10, 2317 (2019).

2. Wang, S. et al. Trophoblast-derived hyaluronan promotes the regulatory phenotype of decidual macrophages. Reproduction 157, 189-198 (2019).

3. Jiang, X., Du, M. R., Li, M. \& Wang, H. Three macrophage subsets are identified in the uterus during early human pregnancy. Cell Mol. Immunol. 15, 1027-1037 (2018).

4. Rheinlander, A., Schraven, B. \& Bommhardt, U. CD45 in human physiology and clinical medicine. Immunol. Lett. 196, 22-32 (2018).

5. Wu, Z., Zhang, Z., Lei, Z. \& Lei, P. CD14: Biology and role in the pathogenesis of disease. Cytokine Growth Factor Rev. 48, 24-31 (2019).

6. Murray, P. J. et al. Macrophage activation and polarization: nomenclature and experimental guidelines. Immunity 41, 14-20 (2014).

7. Beyer, M. et al. High-resolution transcriptome of human macrophages. PLOS ONE 7, e45466 (2012)

8. Wang, X. Q., Zhou, W. J., Hou, X. X., Fu, Q. \& Li, D. J. Trophoblast-derived CXCL16 induces M2 macrophage polarization that in turn inactivates NK cells at the maternal-fetal interface. Cell Mol. Immunol. 15, 1038-1046 (2018).

9. Kim, H. J., Joe, Y., Surh, Y. J. \& Chung, H. T. Metabolic signaling functions of the heme oxygenase/CO system in metabolic diseases. Cell Mol. Immunol. 15, 1085-1087 (2018).

10. Ferreira, A. et al. Sickle hemoglobin confers tolerance to Plasmodium infection. Cell 145, 398-409 (2011).

11. Bach, F. H. Heme oxygenase-1: a therapeutic amplification funnel. FASEB J. 19, 1216-1219 (2005).

12. Gallardo, V., Gonzalez, M., Toledo, F. \& Sobrevia, L. Role of heme oxygenase 1 and human chorionic gonadotropin in pregnancy associated diseases. Biochim Biophys. Acta 1866, 165522 (2020).

13. Arora, N., Sadovsky, Y., Dermody, T. S. \& Coyne, C. B. Microbial vertical transmission during human pregnancy. Cell Host Microbe 21, 561-567 (2017).

14. Wang, R. et al. Tubulin detyrosination promotes human trophoblast syncytium formation. J. Mol. Cell Biol. 11, 967-978 (2019).

15. Wang, S. et al. A case report of neonatal COVID-19 infection in China. Clin Infect Dis, (2020).

16. Gibbings, S. L. et al. Three Unique Interstitial Macrophages in the Murine Lung at Steady State. Am. J. Respir. Cell Mol. Biol. 57, 66-76 (2017). 